\title{
Nonlinear dynamics of a sliding beam on two supports under sinusoidal excitation
}

\author{
R J SOMNAY and R A IBRAHIM \\ Mechanical Engineering Department, Wayne State University, Detroit, MI 48202, \\ USA \\ e-mail: ibrahim@eng.wayne.edu; rjsomnay@yahoo.com
}

\begin{abstract}
This study deals with the nonlinear dynamics associated with large deformation of a beam sliding on two-knife edge supports under external excitation. The beam is referred to as a Gospodnetic-Frisch-Fay beam, after the researchers who reported its static deformation in closed form. The freedom of the beam to slide on its supports imparts a nonlinear characteristic to the force-deflection response. The restoring elastic force of the beam possesses characteristics similar to those of the roll-restoring moment of ships. The Gospodnetic-Frisch-Fay exact solution is given in terms of elliptic functions. A curve fit of the exact solution up to eleventhorder is constructed to establish the governing equation of motion under external excitation. The dynamic stability of the unperturbed beam is examined for the damped and undamped cases. The undamped case reveals periodic orbits and one homoclinic orbit depending on the value of the initial conditions. The response to a sinusoidal excitation at a frequency below the linear natural frequency is numerically estimated for different excitation amplitude and different values of initial conditions covered by the area of the homoclinic orbit. The safe basins of attraction are plotted for different values of excitation amplitude. It is found that the safe region of operation is reduced as the excitation amplitude increases.
\end{abstract}

Keywords. Sliding beam on two supports; Gospodnetic-Frisch-Fay beam; static deformation; force-deflection response; excitation amplitude.

\section{Introduction}

Gospodnetic (1959) presented the static deflection curve of a thin elastic beam forced to deform by three-symmetrical knife-edged supports, which, in the absence of friction, exert forces on the deflected beam. Since the beam is inextensible, there is no limitation on its deflection, and a closed form solution for the deflection curve is given in terms of elliptic functions. The same analysis was documented in Frisch-Fay (1962). It is interesting to observe that the restoring elastic force of the beam is analogous to the roll-restoring moment of ships where it vanishes when the value of the roll angle reaches the capsizing value [see, e.g.,

This paper is dedicated to Prof R N Iyengar of the Indian Institute of Science on the occasion of his formal retirement. 
Roberts (1982) and Moshchuk et al (1995)]. The ship roll-restoring moment is a nonlinear function of the roll angle and exhibits positive slope for the stable roll oscillation and negative slope for the unstable regime.

Recently, the beam has been utilized as a non-traditional isolator by Somnay et al (2005). Nonlinearity becomes important in the study of an isolator system when large deflections occur due to the effects of equipment weight and sustained acceleration. These effects are encountered in the behaviour of suspensions of high-speed vehicles, and mounts for sensitive instruments. Many researchers have conducted studies considering various combinations of restoring force and damping functions. Den Hartog (1931) reported the exact solution for the vibratory response of a symmetric system with both coulomb and viscous damping when subjected to a harmonic forcing function. Hundal \& Parnes (1979) considered the same system when subjected to base excitation. Ravindra \& Mallik (1994) examined the response of vibration isolation system with a cubic hard nonlinear restoring force and combined Coulomb and viscous damping. Ruzicka \& Derby (1971) presented extensive results for isolation systems with linear stiffness and nonlinear $n$th power damping. Kirk (1988) analysed isolators with nonlinear stiffness and linear damping in a random vibration environment.

Another class of flexible extendible beams fall under the broad topic of axially moving solid continua associated with spacecraft antennas. Elmaraghy \& Tabarrok (1975) and Zajaczkowski et al $(1979,1980)$ studied the stability of Euler-Bernoulli beams subjected to periodic sliding motions. These studies were restricted to linear beams; however, the results revealed the complex nature of the instability of sliding beams. Flexible extendible beams have received extensive studies in the area of robotics, specifically in the modelling of flexible links moving through prismatic joints. Vu-Quoc \& Li (1995) presented a comprehensive study of the axially translating beam and employed the so-called geometrically exact beam theory developed originally by Simo \& Vu-Quoc (1986). Behdinan et al (1997) developed the equations of motion for geometrically nonlinear flexible sliding beams, deployed or retrieved through prismatic joints. Behdinan \& Tabarrok (1997) examined the axially rigid sliding beam problem undergoing small deformations is examined. They transformed the boundary conditions to the fixed domain and used Galerkin's approach to study the transient response of the beam. They also extended their approach to the nonlinear, axially inextensible sliding beams undergoing large amplitude vibrations and solve several examples to show the differences between the solutions obtained via linear and nonlinear solvers. Ren et al (1999) studied the nonlinear vibration of a beam with dry friction at the supports. The friction at the interconnected joints imparted the means of energy dissipation to the system. The study utilized numerical simulation to identify the regimes of Coulomb friction coefficient where the beam response would be stable under sinusoidal excitation.

The Gospodnetic-Frisch-Fay beam is very rich in its dynamic characteristics due to the large deformation associated with its motion. The present work is devoted to uncover its nonlinear dynamics in the absence and in the presence of external excitation. The paper begins with the static analysis as reported by Frisch-Fay (1962) and includes the detailed derivation of the closed-form solution of the beam deflection. The unperturbed analysis is then considered for the stability of fixed points and the different regimes of dynamic behaviour associated with different levels of initial energy input. An extensive numerical simulation of the beam response under sinusoidal excitation is carried out to identify the safe basin of attraction. The dependence of the safe domain of attraction on excitation level for different values of excitation frequency reveals that the percentage of safe domain is reduced faster with excitation amplitude for lower values of excitation frequency in the neighborhood of linear resonance. 


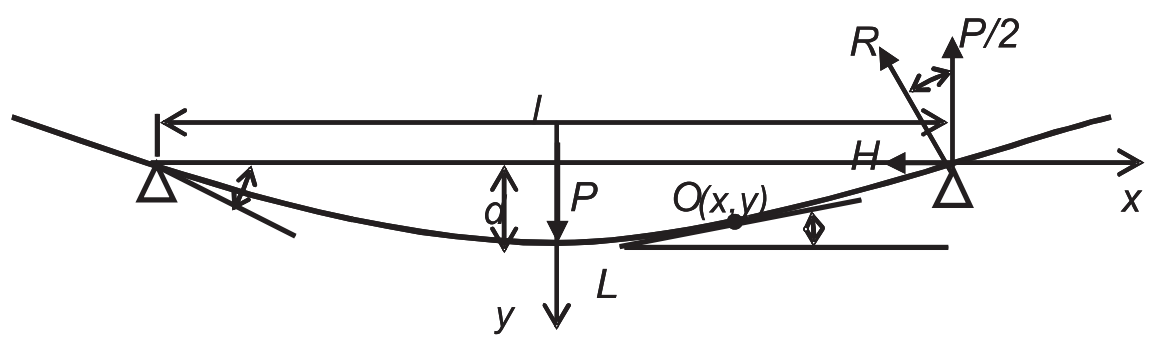

Figure 1. Schematic diagram of deflection under static load of a flexible beam, free to slide at two knife edge supports.

\section{Analytical modelling}

\subsection{Static analysis}

Figure 1 shows a flexible beam which is free to slide at the two knife-edged supports as the applied load $P$ gradually increases. As the load increases, both beam length, $L$, and end slope angle, $\psi_{0}$, increase simultaneously. $d$ denotes the displacement at the mid-span. The two supports A and B are knife-edged supports separated by a distance $l$. Note that $L$ and $l$ are only equal when the beam is horizontal without any sag. The beam deflection as a function of the applied load was originally derived by Gospodnetic (1959) and Frisch-Fay (1962). The deflection of the beam may be written in terms of the slope angle $\psi$ as

$$
\frac{\mathrm{d} \psi}{\mathrm{d} s}=\frac{M}{E I}=\frac{P}{2 E I}\left[(l / 2-x)+\left(y_{\max }-y\right) \tan \psi_{0}\right],
$$

where $M$ is the bending moment. Taking the derivative with respect to $s$, gives

$$
\frac{\mathrm{d}^{2} \psi}{\mathrm{d} s^{2}}=-k^{2}\left[\frac{\mathrm{d} x}{\mathrm{~d} s}+\frac{\mathrm{d} y}{\mathrm{~d} s} \tan \psi_{0}\right]
$$

where $k^{2}=P / 2 E I$. Integrating both sides with respect to $\psi$, gives

$$
\frac{1}{2}\left(\frac{\mathrm{d} \psi}{\mathrm{d} s}\right)^{2}=-k^{2}\left[\sin \psi-\cos \psi \tan \psi_{0}\right]+C .
$$

At $x=l / 2$, the bending moment vanishes, i.e., $(\mathrm{d} \psi / \mathrm{d} s)_{\psi=\psi_{0}}=0$, and thus $C=0$. Accordingly, (3) can be written in the form

$$
\frac{\mathrm{d} \psi}{\mathrm{d} s}=k\left(2\left(\cos \psi \tan \psi_{0}-\sin \psi\right)\right)^{1 / 2}=\sin \psi \frac{\mathrm{d} \psi}{\mathrm{d} y}=\cos \psi \frac{\mathrm{d} \psi}{\mathrm{d} x}
$$

From (4) it is possible to write

$$
\begin{aligned}
& \mathrm{d} x=\cos \psi \mathrm{d} s=\cos \psi\left\{\mathrm{d} \psi / k\left[2\left(\tan \psi_{o} \cos \psi-\sin \psi\right)\right]^{1 / 2}\right\}, \\
& \mathrm{d} y=\sin \psi \mathrm{d} s=\sin \psi\left\{\mathrm{d} \psi / k\left[2\left(\tan \psi_{o} \cos \psi-\sin \psi\right)\right]^{1 / 2}\right\} .
\end{aligned}
$$


Introducing the transformation of variables $\cos \phi=\left[\sin \left(\psi_{o}-\psi\right)\right]^{1 / 2}$ and $\cos \phi_{o}=$ $\left[\sin \psi_{o}\right]^{1 / 2}$, and integrating equations (5) and (6) gives

$$
\begin{aligned}
& l=\left(2 \sqrt{\cos \psi_{o}} / k\right)\left(\sqrt{2} \cos \psi_{o} \cos \phi_{o}+\sin \psi_{o} \Phi\left(p, \phi_{o}\right)\right), \\
& y=\mathrm{d}=\left(\sqrt{\cos \psi_{o}} / k\right)\left(\sqrt{2} \sin \psi_{o} \cos \phi_{o}-\cos \psi \Phi\left(p, \phi_{o}\right)\right),
\end{aligned}
$$

where $\Phi\left(p, \phi_{o}\right)=F\left(p, \phi_{o}\right)-K(p)+2 E(p)-2 E\left(p, \phi_{o}\right)$

$$
=0.8472+F\left(p, \phi_{o}\right)-2 E\left(p, \phi_{o}\right) .
$$

The evaluation of (7) and (8) is given in detail in appendix A.

In order to define the force deflection relationship, (7) and (8) may be written in the form (see appendix A)

$$
\begin{aligned}
P l^{2} / E I & =8 \cos \psi_{o}\left(\sqrt{2} \cos \psi_{o} \cos \phi_{o}+\sin \psi_{o} \Phi\left(p, \phi_{o}\right)\right)^{2}, \\
d / l & =\frac{1}{2} \frac{\sqrt{2} \sin \psi_{o} \cos \phi_{o}-\cos \psi \Phi\left(p, \phi_{o}\right)}{\sqrt{2} \cos \psi_{o} \cos \phi_{o}+\sin \psi_{o} \Phi\left(p, \phi_{o}\right)}
\end{aligned}
$$

The non-dimensional load, $P l^{2} / E I$ and deflection, $d / l$ are plotted as functions of the end slope angle $\psi_{o}$ in figure 2. It shows that by increasing the load the end slope angle is increased and reaches a point where the vertical component of the support reaction is no longer able to support the vertical load. When this happens, the bar slips through. It is seen that the maximum load $\left(P l^{2} / E I\right)_{\max }=6.672$ is reached at the end-slope angle $\psi_{0}=38.3^{\circ}$. For every load less

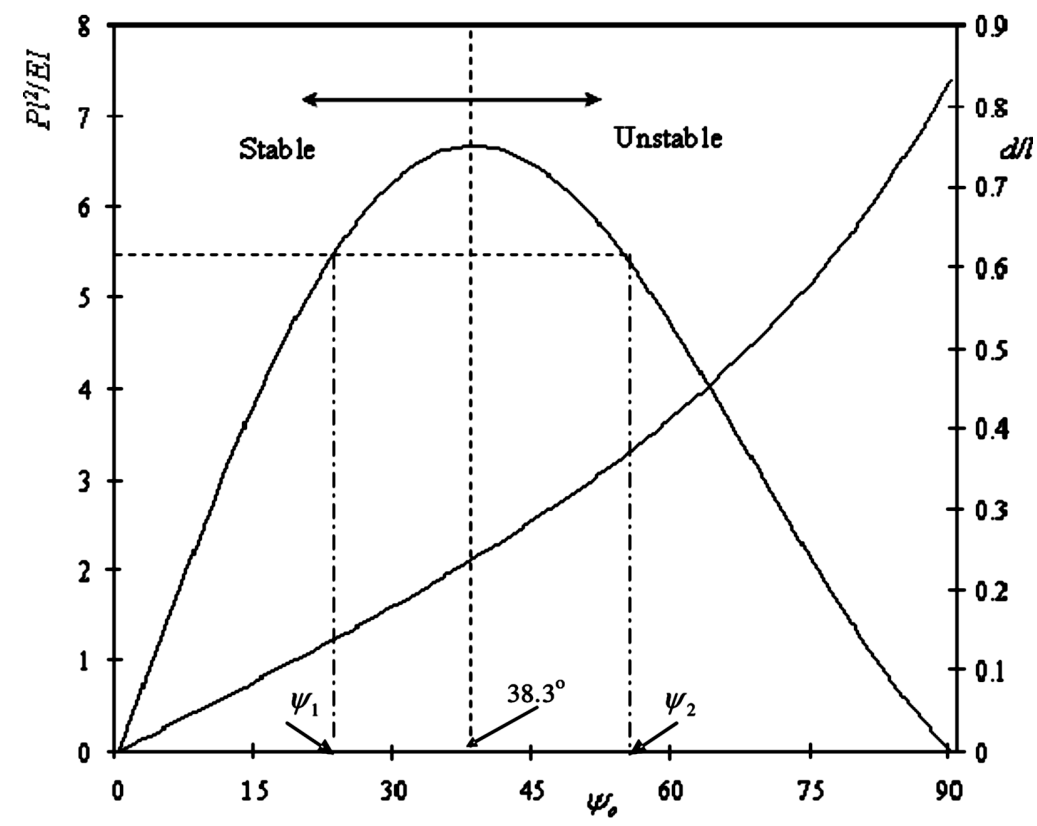

Figure 2. Dependence of load and deflection on end-slope angle based on the exact solution showing the critical angle $\psi_{0_{c r}}=38 \cdot 3^{\circ}$ at which the beam becomes unstable (Gospodnetic 1959). 
than the maximum value there are two different values of the slope angle, $\psi_{01}<38.3^{\circ}$ and $\psi_{02}>38 \cdot 3^{\circ}$. For all values of the slope angle $\psi_{01}<38 \cdot 3^{\circ}$, the beam strain energy is larger than the work done by the static load, and thus the beam is stable. On the other hand, for all values of $\psi_{02}>38.3^{\circ}$ the work done of the static load is larger than the corresponding elastic restoring energy and thus the beam enters into the unstable state. For any load greater than the maximum value $\left(P l^{2} / E I\right)>\left(P l^{2} / E I\right)_{\max }$ the beam becomes unstable and begins to slide and slips off the supports in the absence of friction or constraint forces.

The above analysis provides the load and deflection in closed form in terms of elliptic functions. For the dynamic analysis and to develop the equation of motion, it is convenient to express the load in terms of the beam deflection rather than elliptic functions of the end slope angle. For this reason a polynomial fit is developed. The best fit is found to be of order eleven in the form,

$$
\frac{P l^{2}}{E I}=a_{1}\left(\frac{d}{l}\right)+a_{3}\left(\frac{d}{l}\right)^{3}+a_{5}\left(\frac{d}{l}\right)^{5}+a_{7}\left(\frac{d}{l}\right)^{7}+a_{9}\left(\frac{d}{l}\right)^{9}+a_{11}\left(\frac{d}{l}\right)^{11},
$$

where $a_{1}=48 \cdot 0, a_{3}=-402 \cdot 0, a_{5}=1670 \cdot 6, a_{7}=-3749 \cdot 0, a_{9}=4256 \cdot 4, a_{11}=-1903 \cdot 5$.

Figure 3 shows the plot of the non-dimensional load-deflection curve. The curve exhibits the nonlinear behaviour associated with a soft nonlinearity. At values of deflection over $20 \%$ of the span length there is significant loss of stiffness. Note that this curve is similar to a great extent to the roll-restoring moment of ships. Unlike ship roll motion which possesses two saddle nodes on either side of the zero position, the present system possesses one saddle point on one side of the static equilibrium position as we shall see later.

\subsection{Dynamic modelling}

To simplify the dynamic modelling, the mass of the beam is neglected and the only static load is due to the mass of the carrying load. Consider that the beam carrys a machine of mass, $m$,

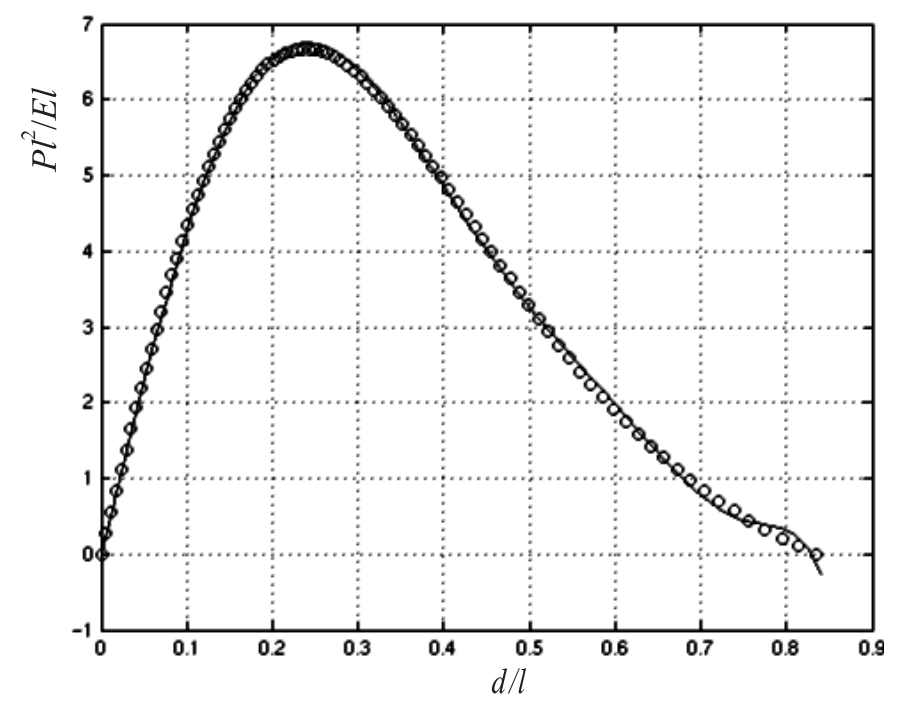

Figure 3. Load deflection curve as generated by an eleventh order polynomial fit compared with the exact solution (o o o: exact solution; $\frac{\sim}{-}$ approximate). 
that produces sinusoidal dynamic unbalance force, $F(t)=F_{0} \sin \Omega t$, where $F_{0}$ and $\Omega$ are the excitation amplitude and frequency respectively. The total potential energy is the sum of gravitational potential energy and elastic potential energy,

$$
\begin{aligned}
V= & \frac{E I d^{2}}{l^{3}}\left(\frac{a_{1}}{2}+\frac{a_{3}}{4}\left(\frac{d}{l}\right)^{2}+\frac{a_{5}}{6}\left(\frac{d}{l}\right)^{4}\right. \\
& \left.+\frac{a_{7}}{8}\left(\frac{d}{l}\right)^{6}+\frac{a_{9}}{10}\left(\frac{d}{l}\right)^{4}+\frac{a_{11}}{12}\left(\frac{d}{l}\right)^{10}\right)-m g d,
\end{aligned}
$$

where $m$ is the mass of the carrying load or machinery. The kinetic energy of the system is given by:

$$
T=\frac{1}{2} m \dot{d}^{2}
$$

The equation for vertical the motion of the system is derived using Lagrange's equation, which yields the following governing equation of motion,

$$
\begin{aligned}
m \ddot{d} & +\frac{E I a_{1}}{l^{3}} d+\frac{E I a_{3}}{l^{5}} d^{3}+\frac{E I a_{5}}{l^{7}} d^{5}+\frac{E I a_{7}}{l^{9}} d^{7}+\frac{E I a_{9}}{l^{11}} d^{9}+\frac{E I a_{11}}{l^{13}} d^{11} \\
& =m g+F(t) .
\end{aligned}
$$

For the development of the dynamic equation of motion it is necessary to modify (14) such that the response displacement is measured from the static equilibrium position. This is done by defining the vibratory or perturbation component of the deflection as $y=d-S$, where $S$ is the static deflection and $d$ is the total deflection. The static component is obtained from the static equilibrium equation:

$$
a_{1} S+\frac{a_{3}}{l^{2}} S^{3}+\frac{a_{5}}{l^{4}} S^{5}+\frac{a_{7}}{l^{6}} S^{7}+\frac{a_{9}}{l^{8}} S^{9}+\frac{a_{11}}{l^{10}} S^{11}=m g \frac{l^{3}}{E I} .
$$

Substituting for $y=d-S$, and (15) into (14) gives the equation of motion in terms of the dynamic displacement, $y$. Introducing the non-dimensional variables, $\tilde{y}=y / l, \tilde{S}=S / l, \tau=$ $\omega_{n} t, f_{o}=F_{0} /\left(m l \omega_{n}^{2}\right)$, and $v=\Omega / \omega_{n},(14)$ takes the form:

$$
\begin{aligned}
\ddot{\tilde{y}}+ & 2 \zeta \dot{\tilde{y}}+\tilde{y}+c_{2} \tilde{y}^{2}+c_{3} \tilde{y}^{3}+c_{4} \tilde{y}^{4}+c_{5} \tilde{y}^{5} \\
& +c_{6} \tilde{y}^{6}+c_{7} \tilde{y}^{7}+c_{8} \tilde{y}^{8}+c_{9} \tilde{y}^{9}+c_{10} \tilde{y}^{10}+c_{11} \tilde{y}^{11}=f_{o} \cos \nu \tau,
\end{aligned}
$$

where $\omega_{n}=\left\{\left(E I / m l^{3}\right)\left[a_{1}+3 a_{3} \tilde{S}^{2}+5 a_{5} \tilde{S}^{4}+7 a_{7} \tilde{S}^{6}+9 a_{9} \tilde{S}^{8}+11 a_{11} \tilde{S}^{10}\right]\right\}^{1 / 2}$ is the linear natural frequency of the beam, and other coefficients are given in appendix B.

\subsection{Stability of fixed points}

Consider the unperturbed beam equation of motion,

$$
\ddot{y}+2 \zeta \dot{y}+F_{11}(y)=0,
$$

where the function $F_{11}(y)=y+c_{2} y^{2}+c_{3} y^{3}+c_{4} y^{4}+\cdots+c_{10} y^{10}+c_{11} y^{11}, c_{2}=-1.9466$, $c_{3}=-7.9378, c_{4}=13.3498, c_{5}=31.8525, c_{6}=-42.3756, c_{7}=-73.9914, c_{8}=$ $63.5792, c_{9}=92.0014, c_{10}=-36 \cdot 2193, c_{11}=-46.8373$, and the tilde has been dropped. 
Equation (17) can be written in the state space form,

$$
\dot{x}_{1}=x_{2}=\dot{y}, \quad \text { and } \quad \dot{x}_{2}=-F_{11}\left(x_{1}\right)-2 \zeta x_{2} .
$$

By setting the left hand-sides of equations (18) to zero, we obtain the following two fixed points,

$$
\left(x_{1}\right)_{1}=0.0 \quad \text { and } \quad\left(x_{1}\right)_{2}=0.446 .
$$

The stability of these fixed points is evaluated by the eigenvalues of the Jacobian matrix:

$$
D_{x} F=\left[\begin{array}{cc}
0 & 1 \\
-F_{11}^{\prime}\left(x_{1}\right) & -(2 \zeta)
\end{array}\right] .
$$

The eigenvalues are given by:

$$
\lambda_{1,2}=-\zeta \pm\left[\zeta^{2}-4 F_{11}^{\prime}\left(x_{1}\right)\right]^{1 / 2} .
$$

For the first fixed point $\left(x_{1}\right)_{1}=0 \cdot 0$, the eigenvalues are $\lambda_{1,2}=-\zeta \pm\left(\zeta^{2}-4\right)^{1 / 2}$. Accordingly, this fixed point is always stable focus point as long as $0<\zeta<2$.

For the second fixed point $\left(x_{1}\right)_{2}=0.446$ the value of $F_{11}^{\prime}=-0.3686$ which represents the slope of the force deflection curve at the point of zero restoring force. The eigenvalues at this point are: $\lambda_{1,2}=-\zeta \pm\left(\zeta^{2}+1.4744\right)^{1 / 2}$. Accordingly, the fixed point is a saddle point for which the system is unstable.

In the absence of damping, the unperturbed motion equation (17) takes the form

$$
\ddot{y}+F_{11}(y)=0 .
$$

From the elastic potential energy, $V(y)=\int_{0}^{y} F\left(y^{\prime}\right) \mathrm{d} y^{\prime}$, and kinetic energy, $T=\frac{1}{2} \dot{y}^{2}$, we can establish the Hamiltonian $H=T+V$ of this system and thus write the first integral of motion,

$$
\dot{y}^{2}=2[H-V(y)] .
$$

As long as $H>V(y)$ the phase diagram is periodic closed orbit in the phase space $\{y, \dot{y}\}$ as shown in figure 4. With reference to figure 5, $H$ reaches its maximum value $H_{\max }=V\left(y_{\max }\right)=$ 0.02365 , where $y_{\max }$ is the maximum beam displacement when its restoring force vanishes. The periodic orbits are only restricted inside the domain $D=\left\{(y, \dot{y}) \mid H \leq H_{c}\right\}$, where $H_{c}=H_{\max }-\Delta H$, and $\Delta H$ is sufficiently small. $H_{c}$ is the critical energy level above which collapse of the beam will take place, and the trajectories of the motion will be structurally unstable. The motion corresponding to $H_{\max }=0.02365$ follows a homoclinic orbit.

Let the system be given an initial velocity $\dot{y}_{0}$, i.e., $H=\dot{y}_{0}^{2} / 2$, The period of oscillation, $T$, can be estimated from (20) as,

$$
T=\frac{1}{\sqrt{2}} \int_{0}^{y} \frac{\mathrm{d} x}{\left[\left(\dot{y}_{0}^{2} / 2\right)-V(x)\right]^{1 / 2}} .
$$

Note that $V(y)<0.02365$ for the entire range of the beam displacement before collapse as shown in figure 5 . The character of motion depends on the value parameter $\left(\dot{y}_{0}^{2} / 2\right)$. For $\dot{y}_{0}^{2} / 2<0.02365$ the integrand is always real and the value of $y$ can assume any value within a range governed by the condition $\left(\dot{y}_{0}^{2} / 2\right)-V(x)=0$. For a given initial energy, $\dot{y}_{0}^{2} / 2<0.02365$, the beam oscillates between two values $y_{1}$ and $y_{2}$ corresponding to the end 


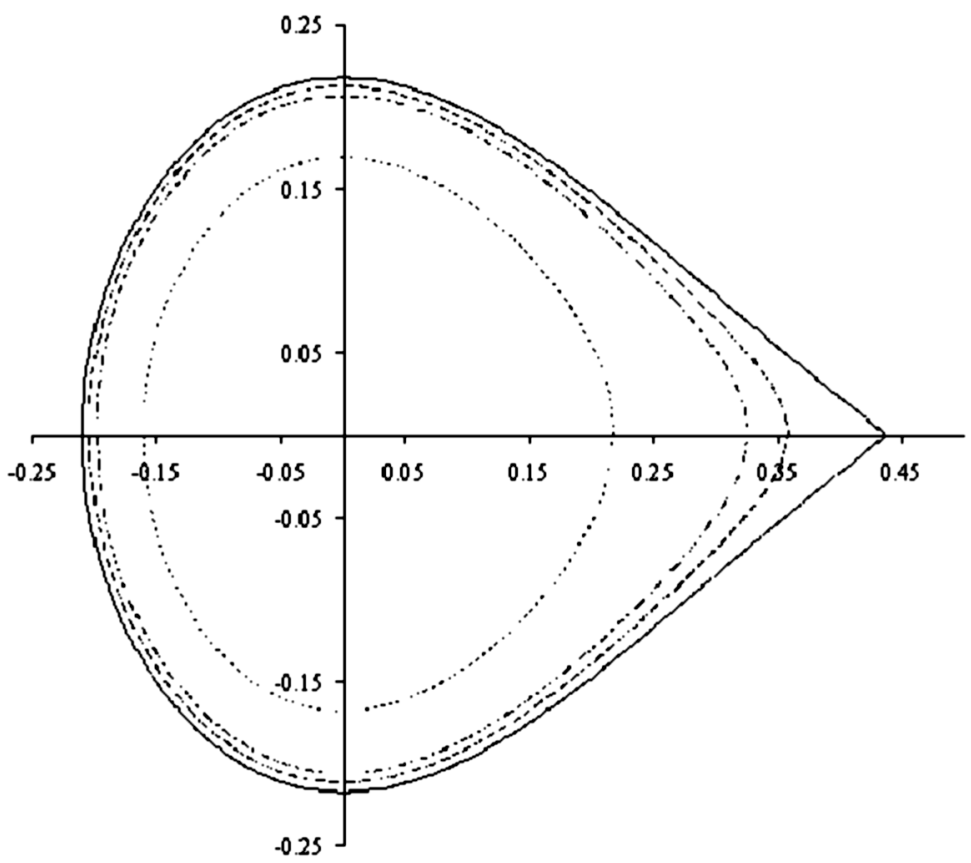

Figure 4. Phase portraits for different levels of initial conditions (energy levels) (....: $H=0 \cdot 01419$; -.-.-.: $H=0.02128 ;---$ : $H=0.02247$; - — $H=0.02365)$.

slope angles $\psi_{01}$ and $\psi_{02}$ respectively, as shown in figure 5. Accordingly the corresponding period of oscillation is

$$
T=\frac{1}{\sqrt{2}} \int_{y_{1}}^{y_{2}} \frac{\mathrm{d} x}{\left[\left(\dot{y}_{0}^{2} / 2\right)-V(x)\right]^{1 / 2}} .
$$

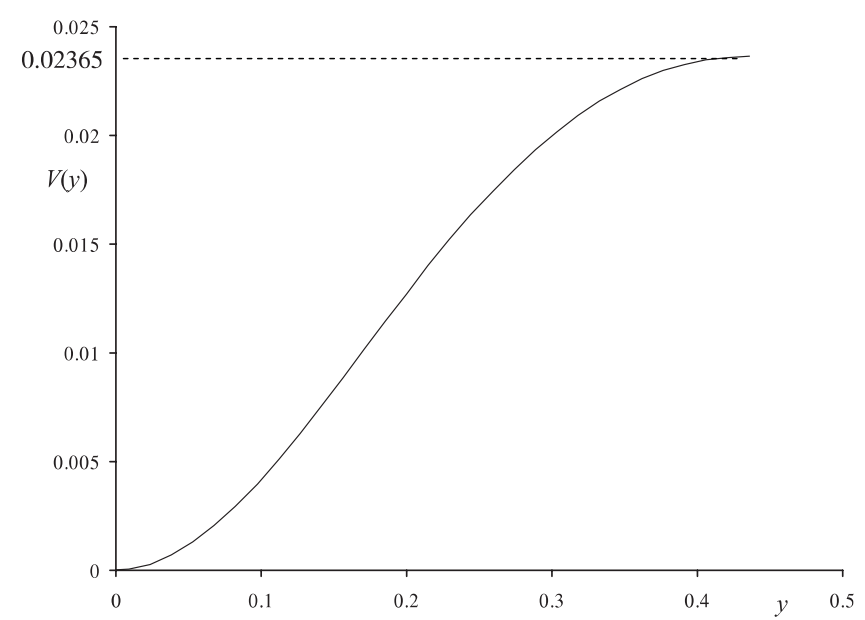

Figure 5. Elastic energy as function of beam displacement. 
If $\dot{y}_{0}^{2} / 2=0.0229$, the integrand is real and approaches $\infty$ and the beam is on the verge of sliding down. If $\dot{y}_{0}^{2} / 2>0.0229$, the integrand is always real and the value of $y$ increases indefinitely. In this case, the motion is unbounded and the beam slides without return.

\subsection{Basin of attraction of perturbed system}

Under forcing excitation, beam response dynamics is governed by the excitation amplitude and frequency and the value of the system inherent damping factor. Depending on the initial conditions the system response may be bounded or grow without limit. The present study is based on numerically integrating the equation of motion (16) for a set of initial conditions covering the entire domain of the phase diagram bounded by the homoclinic orbit. The process is repeated for different values of excitation amplitude and frequency.

Figure 6 shows samples of the safe basins of attraction for different values of excitation amplitude and for excitation frequency parameter $v=0.85$. It is seen that for relatively small values of excitation amplitude, the entire domain bounded by the homoclinic orbit experiences bounded oscillations of period one as shown in figures $6 a$ and $b$. As the excitation amplitude gradually increases, the region is penetrated by a narrow strip leading to unbounded response, where the beam slides continuously without return as shown in figures $6 \mathrm{c}$ and d. Further increase in the excitation amplitude results in more penetrations as shown in figures $6 \mathrm{e}$ and $\mathrm{f}$. To that end, the response in the black region is characterized by bounded oscillations of period one. However, for relatively large excitation amplitude, the response exhibits new features characterized by period doubling and period $n$ for those initial conditions marked by gray spots as shown in figures $6 \mathrm{~g}$ and $\mathrm{h}$. For all excitation levels even slightly larger that $f_{0}=0.041$, the bounded response disappears and the beam becomes completely dynamically unstable for the entire domain of initial conditions. The upper limit of excitation amplitude above which the beam is dynamically unstable is governed by the damping factor and excitation frequency.

Figure 7 shows the dependence of stability fraction, which is estimated by the area of the stable region in the phase plane to the total area encompassed by the homoclinic orbit, on excitation amplitude for three different values of excitation frequency, $v=0.85,0.95$, and 1.15. It is seen that as the excitation frequency increases the upper excitation level increases. This is attributed to the fact that the system is governed by soft nonlinear characteristics and more force is required to produce large response amplitude as excitation frequency increases.

\section{Conclusions}

The vibratory behaviour of a flexible beam without any axial constraint, and therefore free to slide on its supports, has been studied analytically and numerically. A polynomial of the eleventh-order to accommodate the entire range of beam deflection (until it loses its resilient capacity) is developed. The dynamic behaviour of the beam in the absence and the presence of external excitation is studied for the stability of fixed points and response characteristics. For an unperturbed beam, we find that the beam exhibits periodic orbits for all initial conditions that result in an initial energy smaller than the maximum restoring elastic energy. The beam possess a homoclinic orbit for the critical initial energy, equivalent to the maximum restoring elastic energy. Above this energy level the beam slides without return. Under external sinusoidal excitation, safe basins of attractions have been numerically estimated for different values of excitation level. Currently, the random response characteristics of the beam under random excitation is being studied for the response probability density function and other 

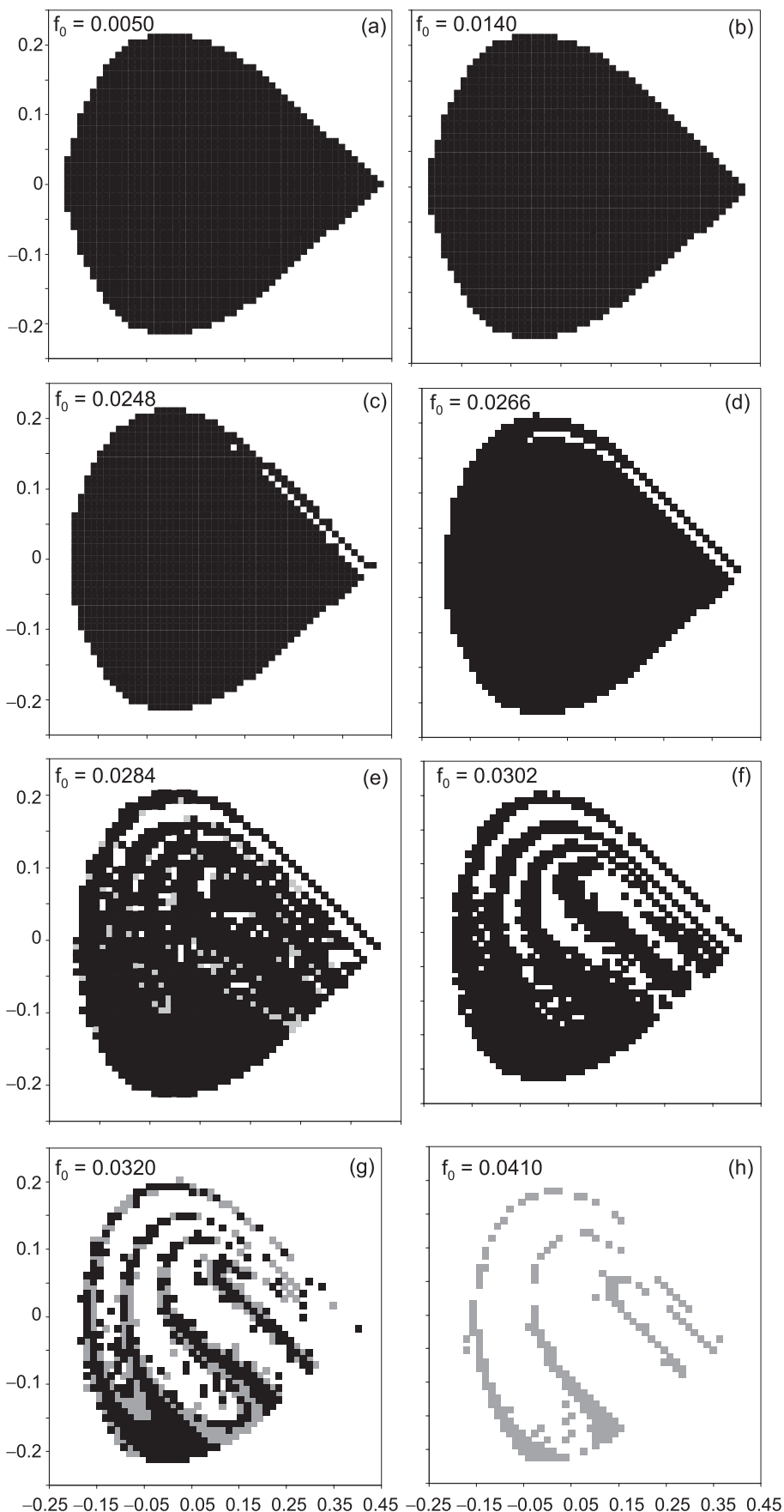

Figure 6. Safe basins of attraction showing different response characteristics for different values of excitation amplitude and excitation frequency, $v=\Omega / \omega_{n}=0.85$. Multi-periodic response; period-one response; empty space: unbounded response. $f_{0}=0.005$ (a), 0.014 (b), 0.0248 (c), 0.0266 (d), 0.0284 (e), 0.0302 (f), 0.032 (g), and 0.041 (h). 


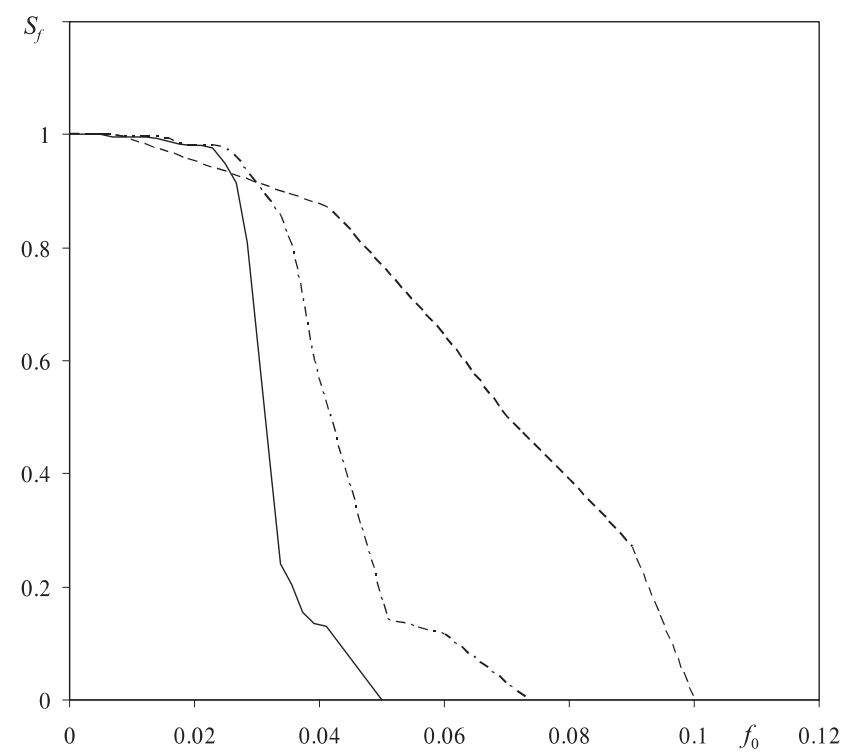

Figure 7. Variation of stability fraction with excitation force amplitude for three different values of excitation frequency. $v=$ $0 \cdot 85$ (- $), 0.95$ (-...-..-...) and $1 \cdot 15(-\ldots)$ - - -

statistical functions using the Fokker-Planck equation. Equally important is the effectiveness of the beam as a nonlinear vibration isolator under deterministic and stochastic excitations, which is also being studied.

\section{Appendix A. Derivation of (7) and (8)}

Starting from the variable transformation

$$
\cos \phi=\left(\sin \left(\psi_{o}-\psi\right)\right)^{1 / 2},
$$

and taking the derivative of both sides gives

$$
\sin \phi \mathrm{d} \phi=\left[\cos \left(\psi_{o}-\psi\right) / 2 \cos \phi\right] \mathrm{d} \psi .
$$

The following identity is used

$$
\cos \left(\psi_{o}-\psi\right)=\left(1-\cos ^{4} \phi\right)^{1 / 2}=\sqrt{2} \sin \phi\left(1-p^{2} \sin ^{2} \phi\right)^{1 / 2},
$$

where $p=1 / \sqrt{2}=\sin (\pi / 4)$.

Substituting (A1) and (A3) into (A2), gives

$$
\mathrm{d} \psi=\left[\sqrt{2} \cos \phi /\left(1-p^{2} \sin ^{2} \phi\right)^{1 / 2}\right] \mathrm{d} \phi .
$$

Note that the limits from $\psi=0$ to $\psi=\psi_{o}$ for the variable $\psi$, correspond to the limits from $\phi=\phi_{o}$, to $\phi=\pi / 2$ respectively. We can also write,

$$
\begin{aligned}
\sin \psi & =\sin \left(\psi_{o}-\left(\psi_{o}-\psi\right)\right)=\sin \psi_{o} \cos \left(\psi_{o}-\psi\right)-\cos \psi_{o} \sin \left(\psi_{o}-\psi\right) \\
& =\sin \psi_{o} \sin \phi \sqrt{2}\left(1-p^{2} \sin ^{2} \phi\right)^{1 / 2}-\cos \psi_{o} \cos ^{2} \phi \\
\cos \psi & =\cos \left(\psi_{o}-\left(\psi_{o}-\psi\right)\right) \\
& =\cos \psi_{o} \sin \phi \sqrt{2}\left(1-p^{2} \sin ^{2} \phi\right)^{1 / 2}+\sin \psi_{o} \cos ^{2} \phi
\end{aligned}
$$


The following integral is needed

$$
\begin{aligned}
\int_{\phi_{o}}^{\pi / 2} \frac{\sin ^{2} \phi \mathrm{d} \phi}{\left(1-p^{2} \sin ^{2} \phi\right)^{1 / 2}}= & \frac{1}{p^{2}} \int_{\phi_{o}}^{\pi / 2} \frac{\left(1-\left(1-p^{2} \sin ^{2} \phi\right) \mathrm{d} \phi\right.}{\left(1-p^{2} \sin ^{2} \phi\right)^{1 / 2}} \\
= & 2\left(\int_{\phi_{o}}^{\pi / 2} \frac{\mathrm{d} \phi}{\left(1-p^{2} \sin ^{2} \phi\right)^{1 / 2}}\right. \\
& \left.-\int_{\phi_{o}}^{\pi / 2}\left(1-p^{2} \sin ^{2} \phi\right)^{1 / 2} \mathrm{~d} \phi\right) \\
= & 2\left[K(p)-F\left(p, \phi_{o}\right)-E(p)+E\left(p, \phi_{o}\right)\right],
\end{aligned}
$$

where

$$
\begin{aligned}
& F\left(p, \phi_{0}\right)=\int_{0}^{\phi_{0}} \frac{\mathrm{d} \varphi}{\left[\left(1-p^{2} \sin ^{2} \phi\right)\right]^{1 / 2}}, \quad \begin{array}{l}
\text { is the incomplete integral } \\
\text { of the first kind }
\end{array} \\
& E\left(p, \phi_{0}\right)=\int_{0}^{\phi_{0}}\left[\left(1-p^{2} \sin ^{2} \phi\right)\right]^{1 / 2} \mathrm{~d} \phi, \quad \begin{array}{l}
\text { is the incomplete integral } \\
\text { of the second kind, }
\end{array} \\
& K(p)=\int_{0}^{\pi / 2} \frac{\mathrm{d} \varphi}{\left[\left(1-p^{2} \sin ^{2} \phi\right)\right]^{1 / 2}} \text {, is the complete integral of the first kind, } \\
& E(p)=\int_{0}^{\pi / 2}\left[\left(1-p^{2} \sin ^{2} \phi\right)\right]^{1 / 2} \mathrm{~d} \phi, \quad \begin{array}{l}
\text { is the complete integral } \\
\text { of the second kind. }
\end{array}
\end{aligned}
$$

Consider the integration of (5)

$$
\begin{aligned}
& \int_{0}^{l / 2} \mathrm{~d} x=\int_{0}^{\psi_{0}} \frac{\cos \psi}{k\left[2\left(\tan \psi_{o} \cos \psi-\sin \psi\right)\right]^{1 / 2}} \mathrm{~d} \psi \\
& \frac{l}{2}=\frac{1}{k} \int_{\phi_{o}}^{\pi / 2} \frac{\sqrt{\cos \psi_{o}}\left(\sqrt{2} \cos \psi_{o} \sin \phi\left(1-p^{2} \sin ^{2} \phi\right)^{1 / 2}+\sin \psi_{o} \cos ^{2} \phi\right)}{\left(1-p^{2} \sin ^{2} \phi\right)^{1 / 2}} \mathrm{~d} \phi \\
& =\frac{\sqrt{\cos \psi_{o}}}{k}\left(\int_{\phi_{o}}^{\pi / 2} \sqrt{2} \cos \psi_{o} \sin \phi \mathrm{d} \phi\right. \\
& \left.+\sin \psi_{o} \int_{\phi_{o}}^{\pi / 2} \frac{1-\sin ^{2} \phi}{\left(1-p^{2} \sin ^{2} \phi\right)^{1 / 2}} \mathrm{~d} \phi\right) \\
& =\frac{\sqrt{\cos \psi_{o}}}{k}\left(\sqrt{2} \cos \psi_{o} \cos \phi_{o}+\sin \psi_{o} \Phi\left(p, \phi_{o}\right)\right) \text {. }
\end{aligned}
$$

The integral $\Phi\left(p, \phi_{o}\right)$ is evaluated by dividing the limits of the integrals into two intervals:

$$
\phi_{o} \rightarrow \pi / 2 \equiv\left(\phi_{o} \rightarrow 0\right)+(0 \rightarrow \pi / 2) \equiv-\left(0 \rightarrow \phi_{o}\right)+(0 \rightarrow \pi / 2) .
$$


This gives:

$$
\begin{aligned}
\Phi\left(p, \phi_{o}\right)= & \int_{\phi_{o}}^{\pi / 2} \frac{1-\sin ^{2} \phi}{\left(1-p^{2} \sin ^{2} \phi\right)^{1 / 2}} \mathrm{~d} \phi \\
= & \int_{0}^{\pi / 2} \frac{\mathrm{d} \phi}{\left(1-p^{2} \sin ^{2} \phi\right)^{1 / 2}} \\
& -\int_{0}^{\phi_{o}} \frac{\mathrm{d} \phi}{\left(1-p^{2} \sin ^{2} \phi\right)^{1 / 2}} \int_{\phi_{o}}^{\pi / 2} \frac{\sin ^{2} \phi \mathrm{d} \phi}{\left(1-p^{2} \sin ^{2} \phi\right)^{1 / 2}} \\
= & K(p)-F\left(p, \phi_{o}\right)-\int_{\phi_{o}}^{\pi / 2} \frac{\sin ^{2} \phi \mathrm{d} \phi}{\left(1-p^{2} \sin ^{2} \phi\right)^{1 / 2}} .
\end{aligned}
$$

The last integral in (A9) is given by (A7). Substituting (A7) into (A9) gives

$$
\begin{aligned}
\Phi\left(p, \phi_{o}\right) & =F\left(p, \phi_{o}\right)-K(p)+2 E(p)-2 E\left(p, \phi_{o}\right) \\
& =0.8472+F\left(p, \phi_{o}\right)-2 E\left(p, \phi_{o}\right) .
\end{aligned}
$$

Substituting for $k=(p / 2 E I)^{1 / 2}$ in (A8) and squaring both sides gives the relationship between load $P$ and end slope angle $\psi_{o}$ in the form,

$$
P l^{2} / E I=8 \cos \psi_{o}\left(\sqrt{2} \cos \psi_{o} \cos \phi_{o}+\sin \psi_{o} \Phi\left(p, \phi_{o}\right)\right)^{2}
$$

A similar procedure is followed to obtain the relationship between the max deflection $d$ at the mid-span and the end slope angle $\psi_{o}$. From equation (6) one writes

$$
\begin{aligned}
\int_{0}^{d} \mathrm{~d} y & =\mathrm{d}=\int_{0}^{\psi_{o}} \frac{\sin \psi \mathrm{d} \psi}{k\left[2\left(\tan \psi_{o} \cos \psi-\sin \psi\right)\right]^{1 / 2}} \\
& =\int_{0}^{\psi_{o}} \frac{\sqrt{\cos \psi_{o}} \cos \psi \mathrm{d} \psi}{k\left[2 \sin \left(\psi_{o}-\psi\right)\right]^{1 / 2}}=\int_{0}^{\psi_{o}} \frac{\sqrt{\cos \psi_{o}} \sin \psi \mathrm{d} \psi}{k \sqrt{2} \cos \phi} \\
& =\frac{1}{k} \int_{\phi_{o}}^{\pi / 2} \frac{\sqrt{\cos \psi_{o}}\left(\sqrt{2} \sin \psi_{o} \sin \phi\left(1-p^{2} \sin ^{2} \phi\right)^{1 / 2}-\cos \psi_{o} \cos ^{2} \phi\right)}{\left(1-p^{2} \sin ^{2} \phi\right)^{1 / 2}} \mathrm{~d} \phi \\
& =\left(\sqrt{\cos \psi_{o}} / k\right)\left(\sqrt{2} \sin \psi_{o} \cos \phi_{o}-\cos \psi \Phi\left(p, \phi_{o}\right)\right) .
\end{aligned}
$$

Alternatively (A11) may be written in the form by substituting for $k l=l(p / 2 E I)^{1 / 2}$,

$$
\frac{d}{l}=\frac{1}{2} \frac{\sqrt{2} \sin \psi_{o} \cos \phi_{o}-\cos \psi \Phi\left(p, \phi_{o}\right)}{\sqrt{2} \cos \psi_{o} \cos \phi_{o}+\sin \psi_{o} \Phi\left(p, \phi_{o}\right)}
$$




\section{Appendix B. Coefficients of (16)}

$$
\begin{aligned}
& c_{2}=\frac{\tilde{S}\left(3 a_{3}+10 a_{5} \tilde{S}^{2}+21 a_{7} \tilde{S}^{4}+36 a_{9} \tilde{S}^{6}+55 a_{11} \tilde{S}^{8}\right)}{\left(a_{1}+3 a_{3} \tilde{S}^{2}+5 a_{5} \tilde{S}^{4}+7 a_{7} \tilde{S}^{6}+9 a_{9} \tilde{S}^{8}+11 a_{11} \tilde{S}^{10}\right)}, \\
& c_{3}=\frac{\left(a_{3}+10 a_{5} \tilde{S}^{2}+35 a_{7} \tilde{S}^{4}+84 a_{11} \tilde{S}^{6}\right)}{\left(a_{1}+3 a_{3} \tilde{S}^{2}+5 a_{5} \tilde{S}^{4}+7 a_{7} \tilde{S}^{6}+9 a_{9} \tilde{S}^{8}+11 a_{11} \tilde{S}^{10}\right)}, \\
& c_{4}=\frac{\tilde{S}\left(5 a_{5} \tilde{S}+35 a_{7} \tilde{S}^{2}+126 a_{9} \tilde{S}^{4}+330 a_{11} \tilde{S}^{6}\right)}{\left(a_{1}+3 a_{3} \tilde{S}^{2}+5 a_{5} \tilde{S}^{4}+7 a_{7} \tilde{S}^{6}+9 a_{9} \tilde{S}^{8}+11 a_{11} \tilde{S}^{10}\right)}, \\
& c_{5}=\frac{\left(a_{5}+21 a_{7} \tilde{S}^{2}+126 a_{9} \tilde{S}^{4}+462 a_{11} \tilde{S}^{6}\right)}{\left(a_{1}+3 a_{3} \tilde{S}^{2}+5 a_{5} \tilde{S}^{4}+7 a_{7} \tilde{S}^{6}+9 a_{9} \tilde{S}^{8}+11 a_{11} \tilde{S}^{10}\right)}, \\
& c_{6}=\frac{\tilde{S}\left(7 a_{7}+84 a_{9} \tilde{S}^{2}+462 a_{11} \tilde{S}^{4}\right)}{\left(a_{1}+3 a_{3} \tilde{S}^{2}+5 a_{5} \tilde{S}^{4}+7 a_{7} \tilde{S}^{6}+9 a_{9} \tilde{S}^{8}+11 a_{11} \tilde{S}^{10}\right)}, \\
& c_{7}=\frac{\left(a_{7}+36 a_{9} \tilde{S}^{2}+330 a_{11} \tilde{S}^{4}\right)}{\left(a_{1}+3 a_{3} \tilde{S}^{2}+5 a_{5} \tilde{S}^{4}+7 a_{7} \tilde{S}^{6}+9 a_{9} \tilde{S}^{8}+11 a_{11} \tilde{S}^{10}\right)}, \\
& c_{8}=\frac{\tilde{S}\left(9 a_{9}+165 a_{11} \tilde{S}^{3}\right)}{\left(a_{1}+3 a_{3} \tilde{S}^{2}+5 a_{5} \tilde{S}^{4}+7 a_{7} \tilde{S}^{6}+9 a_{9} \tilde{S}^{8}+11 a_{11} \tilde{S}^{10}\right)}, \\
& c_{9}=\frac{\left(a_{9}+55 a_{11} \tilde{S}^{2}\right)}{\left(a_{1}+3 a_{3} \tilde{S}^{2}+5 a_{5} \tilde{S}^{4}+7 a_{7} \tilde{S}^{6}+9 a_{9} \tilde{S}^{8}+11 a_{11} \tilde{S}^{10}\right)}, \\
& c_{10}=\frac{11 a_{11} \tilde{S}}{\left(a_{1}+3 a_{3} \tilde{S}^{2}+5 a_{5} \tilde{S}^{4}+7 a_{7} \tilde{S}^{6}+9 a_{9} \tilde{S}^{8}+11 a_{11} \tilde{S}^{10}\right)}, \\
& c_{11}=\frac{a_{11}}{\left(a_{1}+3 a_{3} \tilde{S}^{2}+5 a_{5} \tilde{S}^{4}+7 a_{7} \tilde{S}^{6}+9 a_{9} \tilde{S}^{8}+11 a_{11} \tilde{S}^{10}\right)} \text {. }
\end{aligned}
$$

\section{References}

Behdinan K, Tabarrok B 1997 Dynamics of flexible sliding beams - nonlinear analysis, part II: transient response. J. Sound Vibr. 208: 541-565.

Behdinan K, Stylianou M C, Tabarrok B 1997 Dynamics of flexible sliding beams - nonlinear analysis, part I: formulation. J. Sound Vibr. 208: 517-539

Den Hartog J P 1931 Forced vibrations with constrained coulomb damping and viscous friction. Trans. ASME 53: 107-115

Elmaraghy R, Tabarrok B 1975 On the dynamic stability of an axially oscillating beam. J. Franklin Inst. 30: 25-39

Frisch-Fay R 1962 Flexible beams (Washington, DC: Butterworth)

Gispodnetic D 1959 Deflection curve of a simply supported beam. ASME, J. Appl. Mech. 26: 675-676

Hundal M S, Parnes P S 1979 Response of a base excitation system with Coulomb and viscous friction. J. Sound Vibr. 64: 371-378

Kirk C L 1988 Nonlinear random vibration isolators. J. Sound Vibr. 124: 157-182 
Moshchuk N K, Ibrahim R A, Khasminskii R Z, Chow P 1995 Asymptotic expansion of ship capsizing in random sea waves, part I: First-order approximation. Int. J. Nonlinear Mech. 30: 727-740

Ravindra B, Mallik A K 1994 Performance of nonlinear vibration isolators under harmonic excitation. J. Sound Vibr. 170: 325-337

Ren Y S, Qin H Z, Zhao Z L 1999 Nonlinear vibration analysis of a beam with dry friction at the supports. Mech. Res. Commun. 26: 83-89

Roberts J B 1982 A stochastic theory for nonlinear ship rolling in irregular seas. J. Ship Res. 26: 229-238

Rusicka J E, Derby T F 1971 Influence of damping in vibration isolation (Washington, DC: Shock \& Vibr. Inf. Center)

Simo J C, Vu-Quoc L 1986 The dynamics of flexible beams under large overall motions, the plane case, Part II. ASME J. Appl. Mech. 53: 855-863

Somnay R J, Ibrahim R A, Banasik R 2005 Frisch beam as a nonlinear vibration isolator. Proc. 9th Int. Conf. on Energy and Environment, Sharm-El-Sheik, Egypt

Vu-Quoc L, Li S 1995 Dynamics of sliding geometrically-exact beams: large angle maneuver and parametric resonance. Comput. Meth. Appl. Mech. Eng. 12: 65-118

Zajaczkowski J, Lipinski J 1979 Instability of motion of a beam of periodically varying length. J. Sound Vibr. 63: 9-18

Zajaczkowski J, Yamada G 1980 Further results on instability of the motion of a beam of periodically varying length. J. Sound Vibr. 68: 173-180 